\title{
Localization in Algebraic Field Theory
}

\author{
John E. Roberts \\ Fachbereich Physik, Universität Osnabrück, D-4500 Osnabrück, Federal Republic of Germany
}

\begin{abstract}
The algebra of observables has two distinct local structures. The first, derived from the localization of measurements, gives rise to an additive net structure. The second, derived from the support properties of infinitesimal operations, gives rise to a sheaf structure. It is also shown how an additive net of field algebras acted on by a compact gauge group of the first kind generates an additive net of observable algebras.
\end{abstract}

\section{Introduction}

An elementary particle physicist might be defined as a man who, in face of all the evidence, believes in elementary particles. His more theoretically inclined colleague, the quantum field theorist, is forced to comprehend tracks in bubble chambers and irreducible representations of the Poincare group as compatible aspects of the concept "elementary particle." He will reconcile these aspects using concepts of field theory, which, again in the face of all the evidence, he stubbornly clings to. The ability of "elementary particle" and "field" to survive in the minds of physicists is, in part, their ability to evolve as concepts.

However useful specific quantum fields may prove in analysing specific models, it is unlikely that fields in the sense, say, of unbounded operator-valued distributions, are the basic objects of field theory. Not only are they ill-behaved mathematically but they do not provide an intrinsic description of a physical theory: different fields can describe the same physical theory. It took a man of Rudolf Haag's vision to make a radical break with tradition and provide an alternative view of field theory, algebraic field theory, free of these defects [1]. The basic object is the observable net $\mathfrak{U}$ assigning to a region $\mathcal{O}$ in space-time the algebras $\mathfrak{U}(\mathcal{O})$ generated by the observables which can be measured within $\mathcal{O}$. The connection with fields is that the elements of $\mathfrak{U}(\mathcal{O})$ can be regarded as bounded local functions of the fields, local in the sense that they depend only on the restriction of the fields to $\mathcal{O}$. If fields are introduced at all at this stage, they serve as auxiliary quantities in the construction of the net $\mathfrak{A}$ and one readily understands how different fields can define the same theory. More important is the assertion 
that $\mathfrak{A}(\mathcal{O})$ is generated by observables measurable within $\mathcal{O}$. For all the idealizations involved here, it reestablishes the simple conceptual basis lost in the sophistications of unobservable fields such as the electron field. The implicit claim is that any unobservable fields are already latent in the structure of the observable net. An immediate benefit of the conceptual basis was that local commutativity, $A_{1} A_{2}=A_{2} A_{1}$, if $A_{i} \in \mathfrak{U}\left(\mathcal{O}_{i}\right), i=1,2$, whenever $\mathcal{O}_{1}$ and $\mathcal{O}_{2}$ are spacelike separated, can be linked to Einstein causality since measurements in spacelike separated regions cannot interfere with one another.

In algebraic field theory, the hallmark of a field theory is therefore not a field depending on points in space-time but algebras depending on regions of spacetime. This localization structure is the theme of this paper.

Certain types of operator appear to have two natural localization regions. Here are the standard examples.

a) If there are local charge-carrying fields $\psi$ in the theory, then $\psi(x) \psi(y)^{*}$ is localized around the points $x$ and $y$ although if one wants to express it in terms of observables, the best one can do is to localize it around some path joining $x$ and $y$.

b) If a charge is associated with a conserved current $j^{\mu}$ then $Q_{r}=\int_{|\mathbf{x}| \leqq r} j^{0}(0, \mathbf{x}) d \mathbf{x}$ is localized around the ball $|\mathbf{x}| \leqq r$. Judged by its commutation relations with the observable net, $Q_{r}$ appears to be localized around the sphere $|\mathbf{x}|=r$.

c) The flux $\Phi(S)$ of the magnetic field through a surface $S$ is obviously localized around $S$ but, since it depends only on the boundary $\partial S$ of $S$, it might be considered as being localized around $\partial S$ instead.

These examples have certain common features. The smaller localization region has a non-trivial homotopy invariant: in a) it is disconnected, in c) it is not simply connected and in b) it is not 2-connected. These non-trivial homotopy invariants are closely linked with non-trivial homological invariants and the specific operators which appear suggest very strongly that some form of homology and cohomology is involved. In c) it is a 2-cohomology, in a) a 1-cohomology and in b) a 1-homology. The relation of these examples with gauge groups is also significant. a) and b) relate to a gauge group of the first kind. Indeed in the case of an electric charge, where one has a gauge group of the second kind, $\psi(x) \psi(y)^{*}$ should be replaced by $\psi(x) \exp \left(i e \int A^{\mu}\left(x^{\prime}\right) d b_{\mu}\left(x^{\prime}\right)\right) \psi(y)^{*}$ which depends explicitly on a path $b$, and $j^{\mu}=\partial_{v} F^{\mu \nu}$ so that the ambiguity in localization is again resolved, this time in favour of the smaller region. c) on the other hand is typical of gauge theories.

I learned a large part of these arguments from Rudolf Haag more than ten years ago and they have motivated most of my research during the past five years.

Another interesting feature of these examples becomes apparent on examining the role of the operators involved. Although one talks about the net $\mathfrak{A}$ of observables, the role that the elements of $\mathfrak{A}$ play in practice is frequently not that of an observable. Instead it is their action on state vectors, hence on states, that is important, in which case it is usual to speak of an operation rather than an observable. In particular, whenever we deal with a unitary $V$ of $\mathfrak{A}$, its physical role is likely to be that of an operation $\omega \rightarrow \omega \circ \mathrm{ad} V$. However, even a self-adjoint operator $A$ of $\mathfrak{A}$ may play the role of an infinitesimal operation: it generates the 1 -parameter group of operations $\omega \rightarrow \omega \circ \mathrm{ad} e^{i \lambda A}$. Of course not all interesting operations can be described by the action of elements of $\mathfrak{A}$. 
Turning to our examples again, in a) $\psi(x) \psi(y)^{*}$ plays the role of an operation: charge is transferred from $y$ to $x$. In b) $Q_{r}$ is an observable, the charge confined within $|\mathbf{x}| \leqq r$ at $x^{0}=0 . \Phi(S)$ in c) is particularly instructive. On the face of it, it is an observable, the flux of the magnetic field through $S$. This refers, however, to our way of describing the operator. In practice, it may be the operation $e^{i \Phi(S)}$ which is involved. The physical interpretation is quite different: $e^{i \Phi(S)}$ creates an electric field localized on $\partial S$ at $t=0$. Thus we must distinguish carefully between these two aspects and the two different localization structures which emerge are described in this paper. The other interesting facets of these examples can merely be commended to the reader's attention.

The localization of observables is treated conventionally with the emphasis in Sect. 1 being placed on additivity as the basic property of the associated net structure. Additivity in space is shown to be related to additivity in space-time through the causal propagation character of the theory. The main result, however, is that additivity survives the transition from fields to observables in the presence of a compact gauge group of the first kind. Thus additivity can be regarded as well established for the current range of models.

The localization of infinitesimal operations is treated unconventionally, with attention in Sect. 2 being focussed not on the localization regions but on the regions where they act trivially. Thus $S=S^{*} \in \mathfrak{Q}$ corresponds to an infinitesimal operation $A \rightarrow i[S, A]$ which acts trivially on $\mathcal{O}$ if $[S, A]=0, A \in \mathfrak{A}(\mathcal{O})$. In this way, we are led to the $C^{*}$-algebras $\mathscr{S}(\mathcal{O})$ where

$$
\mathscr{S}(\mathcal{O})=\mathfrak{A}(\mathcal{O})^{c},
$$

the relative commutant of $\mathfrak{A}(\mathcal{O})$. As a consequence of additivity we have

$$
\mathscr{S}\left(\bigcup_{i} \mathcal{O}_{i}\right)=\bigcap_{i} \mathscr{S}\left(\mathcal{O}_{i}\right) .
$$

This means that $\mathscr{S}$ can be made into a sheaf of $C^{*}$-algebras by modifying the definition on the empty set and on disconnected sets. The technical details can be found in [2, Sect. 5] but play no role here. The presence of an underlying sheaf promises to be of importance to the future development of the theory. Minkowski space is now equipped with a sheaf of $C^{*}$-algebras and can be regarded as defining a quantum geometry. This intuition is supported by the preliminary results in $[2,3]$ since it leads naturally to the structures relating to unobservable fields and superselection sectors.

Under these circumstances, one naturally asks whether the sheaf structure determines the net structure. This will, in particular, be the case if

$$
\mathfrak{A}(\mathcal{O})=\mathscr{S}(\mathcal{O})^{c}
$$

for some base for the topology of Minkowski space. The equality in (0.3) is termed internal duality and is a consequence of duality for the region in question but can also hold when duality fails.

The paper concludes with an appendix giving useful results of a more technical nature relating to the action of a compact gauge group commuting with spacetime translations on an additive field net. 


\section{Additivity}

The property of additivity, that the observable algebra of an open set is generated by the observable algebras of any open covering of that set, deserves attention in any study of localization. Exalted to a principle, it provides a means of avoiding ambiguities in localization of the type discussed in the introduction, since the topology of Minkowski space has a base consisting of contractible sets. Viewed in terms of measurement, such a principle is at best inaccessible, at worst implausible. Perhaps it can best be interpreted as a claim that the basic dynamical variables can be arbitrarily well localized.

To formulate additivity precisely we must specify the topology involved. The question of whether additivity holds in the norm topology is premature since the local $C^{*}$-algebras remain elusive in models. The CCR algebras, for example, have the grave defect, albeit not shared by the CAR algebras, that they depend sensitively on the relatively arbitrary choice of test functions and would certainly not yield nets which are additive as $C^{*}$-algebras. Another natural topology would be the weak topology determined by the states of physical interest (to elementary particle physics). This runs into a similar problem in that the states of physical interest can also be elusive in models, although Buchholz and Fredenhagen [4] have come at least close to providing a definitive answer for theories with a mass gap. Fortunately, the restriction of this weak topology to the algebras of bounded open sets or, more precisely, the net $\mathfrak{R}$ of local von Neumann algebras over the set $\mathscr{B}$ of bounded open sets seems to be readily accessible in models. In fact, it usually suffices to pick any candidate for the net $\mathfrak{A}$ of $C^{*}$-algebras over $\mathscr{B}$ and take the weak closure of $\mathfrak{A}(\mathcal{O})$ in the vacuum representation.

Hence, in this section, we shall work exclusively with nets of von Neumann algebras, $\mathfrak{R}$ for the observable net and $\mathfrak{F}$ for the field net. All states and representations of these nets considered will be supposed locally normal. For any net $\mathfrak{R}, C^{*}(\Re)$ denotes the $C^{*}$-inductive limit over the net. A net $\Re$ of von Neumann algebras over some set of open sets will be said to be additive if

$$
\mathfrak{R}(\mathcal{O})=\bigvee_{i} \mathfrak{R}\left(\mathcal{O}_{i}\right) \text { whenever } \quad \mathcal{O}=\bigcup_{i} \mathcal{O}_{i},
$$

i.e. if the $\mathfrak{R}\left(\mathcal{O}_{i}\right)$ generate $\mathfrak{R}(\mathcal{O})$ as a von Neumann algebra. Note that an additive net over the set $\mathscr{K}$ of open double cones has a unique extension to an additive net over $\mathscr{B}$.

We first discuss the relation between additivity in space-time and additivity in space. As is probably clear, we can pass from one to the other by invoking the causal propagation character of the theory. In the one direction, we can express this by requiring of a net $\mathfrak{F}$ over $\mathscr{B}$ that

$$
\mathfrak{F}(Z)=\mathfrak{F}\left(Z^{\prime \prime}\right)
$$

for each bounded open cylinder $Z$ with timelike axis. This property was introduced in [5] and discussed more recently in the context of generalized free fields in [6]. The spacelike part of the extension involved in (1.1) is actually a theorem [7]. 
1.1. Proposition. Let $\mathfrak{F}$ be an additive net over $\mathscr{B}$ satisfying (1.1). Let $H$ be a spacelike hyperplane, then the net $\mathfrak{F}_{H}$ defined by $\mathfrak{\mho}_{H}(B)=\mathfrak{F}(C(B))$, where $B$ is a bounded open set of $H$ and $C(B)$ is the double cone based on $B$, is additive.

Proof. We must show that $B=\bigcup_{i} B_{i}$ implies $\mathfrak{F}(C(B))=\bigvee_{i} \mathfrak{F}\left(C\left(B_{i}\right)\right)$. Without loss of generality, we may suppose the $B_{i}$ to be open balls and $H$ to be the hyperplane $x^{0}=0$. Let $\mathscr{Z}$ denote the set of bounded open cylinders $Z$ with $Z \subset \bigcup_{i} C\left(B_{i}\right)$. One checks easily that $\cup\left\{Z^{\prime \prime}: Z \in \mathscr{Z}\right\}=C(B)$, so that $\mathfrak{F}(C(B))=\vee\{\mathfrak{F}(Z): Z \in \mathscr{Z}\}$ completing the proof.

Note that we should still have such nets $\mathfrak{F}_{H}$ even when the sharp-time Wightman fields do not exist. The converse step just relies on the finite propagation speed as used by Glimm and Jaffe [8] when constructing algebras in space-time from algebras at time zero.

1.2. Proposition. Let $\mathfrak{F}_{0}$ be an additive net over open balls in space and $\alpha$ a 1-parameter group of locally normal automorphisms of $\bigcup_{B} \mathfrak{F}_{0}(B)$ of propagation speed $c=1$, then there is a unique additive net $\mathfrak{F}$ over $\mathscr{B}$ with

$$
\mathfrak{F}(C(B)+t)=\alpha_{t}\left(\mathfrak{F}_{0}(B)\right)
$$

for each open ball $B$ and $t \in \mathbb{R}^{1}$.

Proof. Open sets of the form $C(B)+t$ are a base for the topology of Minkowski space and $\alpha_{t}$ is locally normal so it suffices to show that $C(B)=\bigcup_{i}\left(C\left(B_{i}\right)+t_{i}\right)$ implies $\mathfrak{F}_{0}(B)=\bigvee_{i} \alpha_{t_{i}}\left(\mathfrak{F}_{0}\left(B_{i}\right)\right)$. However there are open balls $\hat{B}_{j}$ with $B=\bigcup_{j} \hat{B}_{j}$ and $\hat{B}_{j}+\left\{\mathbf{x}:|x|<\left|t_{i}\right|\right\} \subset B_{i}$ for some $i=i(j)$. Now finite propagation speed implies $\mathfrak{F}_{0}\left(\hat{B}_{j}\right) \subset \alpha_{t_{i}}\left(\mathfrak{F}_{0}\left(B_{i}\right)\right)$ for $i=i(j)$ so the result follows from the additivity of $\mathfrak{F}_{0}$.

In the above setting, there is a standard route for proving (1.1). If $Z$ has axis in the time direction, it follows from the spectrum condition and [7], so Lorentz covariance would complete the proof.

We now relate additivity for observable nets to additivity for field nets using the following hypotheses:

a) $\mathfrak{F}$ is an additive field net of von Neumann algebras over $\mathscr{B}$ given in a faithful factorial representation on a separable Hilbert space $\mathscr{H}$.

b) There is a continuous unitary representation of space-time translations on $\mathscr{H}$ satisfying the spectrum condition and inducing an action $\alpha$ on $\mathfrak{F}$

$$
\alpha_{x}(\mathfrak{F}(\mathcal{O}))=\mathfrak{F}(\mathcal{O}+x), \mathcal{O} \in \mathscr{B} .
$$

c) $\alpha$ commutes with a compact group $\mathscr{G} \subset$ Aut $\mathfrak{F}$

$$
g(\mathfrak{F}(\mathcal{O}))=\mathfrak{F}(\mathcal{O}), g \in \mathscr{G}, \mathcal{O} \in \mathscr{B} .
$$

d) $\mathfrak{F}$ has Bose-Fermi commutation relations and $\mathscr{G}$ contains the involution $k$ which changes the sign of Fermi fields leaving Bose fields invariant. $k$ is automatically in the centre of $\mathscr{G}$.

Under these circumstances we have 
1.3. Theorem. The net $\mathfrak{R}$ of von Neumann algebras defined by

$$
\mathfrak{R}(\mathcal{O})=\{A \in \mathfrak{F}(\mathcal{O}): g A=A, g \in \mathscr{G}\}
$$

for $\mathfrak{O} \in \mathscr{B}$ connected and

$$
\mathfrak{R}(\mathcal{O})=\bigvee_{i} \mathfrak{R}\left(\mathcal{O}_{i}\right)
$$

if the $\mathcal{O}_{i}$ are the connected components of $\mathcal{O}$ is additive.

To prove the result, we need detailed information on the action of the gauge group given as Theorem A.2 of the appendix whose proof involves additivity in one essential way: if $\mathcal{O}$ is non-empty and

$$
g F=F, F \in \mathfrak{F}(\mathcal{O}),
$$

a) and b) imply that $g$ is the identity automorphism of $\mathfrak{F}$.

The remaining steps in the proof of Theorem 1.3 are contained in

1.4. Lemma. Let $\mathcal{O}, \mathcal{O}_{i} \in \mathscr{B}$ be non-empty and connected, $i \in I$ with $\mathcal{O}=\bigcup_{i \in I} \mathcal{O}_{i}$. Given $i_{0}$, $i^{\prime} \in I$ and a separable $\mathscr{G}$-invariant Hilbert space $H$ in $\mathfrak{F}\left(\mathcal{O}_{i^{\prime}}\right)[9]$, let $M=\bigvee_{i \in I} \mathfrak{R}\left(\mathcal{O}_{i}\right)$ then

a) there exist $i_{1}, i_{2}, \ldots, i_{n}=i^{\prime} \in I$ with $\mathcal{O}_{i_{j-1}} \cap \mathcal{O}_{i_{j}} \neq \emptyset, j=1,2, \ldots, n$,

b) there is a unitary $V \in M$ with $V H \subset \mathbb{F}\left(\mathcal{O}_{i_{0}}\right)$,

c) the endomorphism @ of $\mathfrak{F}$ defined uniquely by

$$
\psi F=\varrho(F) \psi, \psi \in H, F \in \mathfrak{F}
$$

satisfies $\varrho(M) \subset M$.

Proof. Let $I^{\prime}$ denote the subset of elements of $I$ which can be joined by a finite chain to $i_{0}$ as in a). Then $\bigcup_{i \in I^{\prime}} \mathcal{O}_{i} \cap \bigcup_{i \neq I^{\prime}} \mathcal{O}_{i}=\emptyset$. Since $\mathcal{O}$ is connected and each $\mathcal{O}_{i}$ is non-empty and open, $I^{\prime}=I$ proving a). If $\mathcal{O}_{i_{0}} \cap \mathcal{O}_{i^{\prime}} \neq \emptyset$, there is a $\mathscr{G}$-invariant Hilbert space in $\mathfrak{F}\left(\mathcal{O}_{i_{0}} \cap \mathcal{O}_{i^{\prime}}\right)$ equivalent to $H$ as a $\mathscr{G}$-module by Theorem A.2. Hence there is a unitary $V \in \mathfrak{R}\left(\mathcal{O}_{i^{\prime}}\right)$ with $V H \subset \mathfrak{F}\left(\mathcal{O}_{i_{0}}\right)$. b) now follows from a). Obviously, $\varrho\left(\mathfrak{R}\left(\mathcal{O}_{i^{\prime}}\right)\right) \subset \mathfrak{R}\left(\mathcal{O}_{i^{\prime}}\right)$. But by b), $\varrho\left(\mathfrak{R}\left(\mathcal{O}_{i_{0}}\right)\right) \subset V^{*} \mathfrak{R}\left(\mathcal{O}_{i_{0}}\right) V \subset M$ proving c).

Proof of Theorem 1.3. It suffices to show that if $\mathcal{O}=\bigcup_{i \in I} \mathcal{O}_{i}$ as in Lemma 1.4, $\mathfrak{R}(\mathcal{O})=\bigvee_{i \in I} \mathfrak{R}\left(\mathcal{O}_{i}\right)$. By Theorem A.2 and standard harmonic analysis on a compact group, finite sums $\sum_{j=1}^{n} A_{j} \psi_{j}$ with $A_{j} \in \mathfrak{R}(\mathcal{O})$ and $\psi_{i}$ in some finite-dimensional $\mathscr{G}$-invariant Hilbert space in $\mathfrak{F}(\mathcal{O})$ form a weakly dense $*$-subalgebra of $\mathfrak{F}(\mathcal{O})$. Since $\mathfrak{F}(\mathcal{O})=\bigvee_{i} \mathfrak{F}\left(\mathcal{O}_{i}\right)$, Lemma 1.4 shows that we may, in addition, require $A_{j} \in \bigvee_{i} R\left(\mathcal{O}_{i}\right)$ and $\psi_{j} \in \mathfrak{F}\left(\mathcal{O}_{i^{\prime}}\right)$. Averaging these sums over $\mathscr{G}$, a weakly continuous operation, now gives the required result.

Thus with a compact gauge group of the first kind, additivity survives the passage from fields to observables provided we take care to define the observable algebras of disconnected regions correctly. If we take $\mathfrak{F}$ to be the net generated by the free scalar field $\phi$ of mass zero with the spontaneously broken gauge symmetry $\phi(x) \rightarrow \phi(x)+a$, then $\mathfrak{R}$ is the net generated by $\partial^{\mu} \phi$ and we have a simple example of 
the above phenomenon where $\mathscr{G}=\mathbb{R}$ is no longer compact. The question of what happens in gauge theories is obviously premature although example c) of the introduction indicates that care is needed when regions are not simply connected.

We conclude this section by remarking that of course proving additivity for field nets presents no particular problems in practice. After all, additivity had its origins in Wightman field theory. If $\mathcal{O}=\bigcup_{i} \mathcal{O}_{i}$ and $f$ is a test function with support in $\mathcal{O}$, then using a partition of the identity, we can write $f=\sum_{i} f_{i}$, where $f_{i}$ has its support in $\mathcal{O}_{i}$. Hence if $\phi$ is a Wightman field $\phi(f)=\sum_{i} \phi\left(f_{i}\right)$ and additivity for the net generated by the fields follows modulo problems of essential self-adjointness. In particular, for free Bose fields, where we could also argue in terms of Weyl operators, or free Fermi fields additivity is satisfied. For $P(\phi)_{2}$ theories, additivity would follow from local normality and Proposition 1.2. More generally, we could, for example, invoke Corollary 2.5 of [10] and the remarks following it to deal with a class of theories derived from Euclidean Green's functions.

\section{Sheaf Structures}

The local $C^{*}$-algebras, although still elusive in models, may well turn out to carry information which gets lost at the level of the local von Neumann algebras. For this reason, we shall work here with a net $\mathfrak{U}$ of $C^{*}$-algebras of observables over $\mathscr{B}$ but take care to link it to the more accessible additive net $\mathfrak{R}$ of local von Neumann algebras over $\mathscr{B}$ by supposing that $\mathfrak{U}(\mathcal{O})=C^{*}(\mathfrak{U}) \cap \mathfrak{R}(\mathcal{O})$ and is weakly dense in $\mathfrak{R}(\mathcal{O})$. For example, $\mathfrak{A}(\mathcal{O})$ might be the $C^{*}$-subalgebra of $\mathfrak{R}(\mathcal{O})$ whose elements have norm continuous orbits under the Poincare group or the translation group (cf. [11, Satz 9.2]).

As discussed in the introduction, self-adjoint elements of $C^{*}(\mathfrak{U})$ play a dual role as observables and as infinitesimal operations. We investigate here a local structure on $C^{*}(\mathfrak{U})$ determined by the support properties of these infinitesimal operations. If $S \in C^{*}(\mathfrak{U})$ and $U$ is an open, but not necessarily bounded, set we write

$$
S \in \mathscr{S}(U) \Leftrightarrow S A=A S, A \in \mathfrak{U} \mid U
$$

Here $A \in \mathfrak{A} \mid U$ means $A \in \mathfrak{U}(\mathcal{O})$ for some $\mathscr{O} \in \mathscr{B}$ with $\mathcal{O} \subset U$. In other words, $\mathscr{S}(U)=(\mathfrak{A} \mid U)^{c}$, the relative commutant of $\mathfrak{A} \mid U$ in $C^{*}(\mathfrak{A}) . \mathscr{S}(U)$ is a $C^{*}$-algebra and, as an expression of locality,

$$
\mathfrak{U} \mid U^{\prime} \subset \mathscr{S}(U) .
$$

If $V \subset U$, we obviously have $\mathscr{S}(U) \subset \mathscr{S}(V)$. However, as explained in the introduction, what is interesting is the following simple result which shows that $\mathscr{S}$ is essentially a sheaf.

$$
\text { 2.1. Theorem. } \mathscr{S}\left(\bigcup_{i} U_{i}\right)=\bigcap_{i} \mathscr{S}\left(U_{i}\right) \text {. }
$$

Proof. Pick a faithful locally normal representation of $C^{*}(\mathfrak{R})$. The existence of such a representation, in practice evident, is guaranteed by [12, Sect. 3]. Since $\mathfrak{U}(\mathcal{O})$ is 
weakly dense in $\mathfrak{R}(\mathcal{O}), \mathscr{S}(U)=(\mathfrak{R} \mid U)^{c} \cap C^{*}(\mathfrak{U})$, where $C^{*}(\mathfrak{U})$ is identified with a subalgebra of $C^{*}(\mathfrak{R})$. We may now suppose without loss of generality that $\mathfrak{A}=\mathfrak{R}$. If $\mathscr{O} \subset \bigcup_{i} U_{i}, \mathcal{O}=\bigcup_{i}\left(\mathcal{O} \cap U_{i}\right)$, so given $S \in \bigcap_{i} \mathscr{S}\left(U_{i}\right)$ and $A \in \mathfrak{R}(\mathcal{O})$, additivity for $\mathfrak{R}$ implies $S A=A S$.

This result shows that $\mathscr{S}$ like $\mathfrak{U}$ is determined by its values on small open sets. We remark that if $\mathfrak{R}$ satisfies (1.1) then evidently

$$
\mathscr{S}(Z)=\mathscr{S}\left(Z^{\prime \prime}\right)
$$

for each bounded open cylinder with timelike axis. Hence the corresponding sheaf resembles the sheaves of holomorphic functions in that there is an analogue of domains of holomorphy. Proposition 1.2 obviously gives information on the restriction of the sheaf to spacelike hyperplanes but we refrain from giving details.

One of the interesting questions now arising is whether the sheaf structure on $C^{*}(\mathfrak{U})$ also determines the net structure. The following concept is both relevant and beguilingly simple: we say that internal duality holds for some $\mathcal{O} \in \mathscr{B}$ if

$$
\mathfrak{U}(\mathcal{O})=\mathscr{S}(\mathcal{O})^{c} \text {. }
$$

Note that, unlike duality, internal duality is representation independent. Obviously, if internal duality holds for some base for the topology of Minkowski space, e.g. for double cones, then the sheaf structure on $C^{*}(\mathfrak{H})$ determines the net structure.

2.2. Proposition. Suppose duality holds for $\mathcal{O}$ in some faithful locally normal representation $\pi$ of $\mathfrak{R}$, then internal duality holds for $\mathcal{O}$.

Proof. Since $\mathfrak{X} \mid \mathcal{O}^{\prime} \subset \mathscr{S}(\mathcal{O}), \pi(\mathfrak{R}(\mathcal{O}))=\pi\left(\mathfrak{R}\left(\mathcal{O}^{\prime}\right)\right)^{\prime} \cap \pi(\mathfrak{R}) \supset \pi\left(\mathscr{S}(\mathcal{O})^{c}\right)$. But $\pi$ is faithful so $\mathscr{S}(\mathcal{O})^{c} \subset \mathfrak{R}(\mathcal{O}) \cap C^{*}(\mathfrak{U})=\mathfrak{U}(\mathcal{O})$ as required.

Now we can only deduce duality for $\mathfrak{R}$ from twisted duality for $\mathfrak{F}$ for gauge groups that are not spontaneously broken. In the case of internal duality we can do better: for our purposes, with Bose-Fermi commutation relations d), the twisted field net $\mathfrak{F}^{t}$ can be defined using [13, (4.7)]. Any covariant representation $\pi$ of $\{\mathfrak{F}, k\}$ has a canonical extension to the covariance algebra of $\{\mathfrak{F}, k\}$ and hence to $\mathcal{F}^{t}$. Twisted duality is said to hold for $\mathcal{O}$ in $\pi$ if

$$
\pi\left(\mathfrak{F}^{t} \mid \mathcal{O}^{\prime}\right)^{\prime} \cap \pi(\mathfrak{F})=\pi(\mathfrak{F}(\mathcal{O}))
$$

We now have

2.3. Proposition. Let $\mathfrak{F}$ be a field net satisfying a)-d) with twisted duality holding in some (faithful) locally normal covariant representation of $\{\mathfrak{F}, k\}$ for some connected $\mathcal{O} \in \mathscr{B}$. Let $\mathfrak{R}$ be the additive net defined in Theorem 1.3. Then $\mathfrak{A}$ satisfies internal duality for $\mathcal{O}$ if either $\mathcal{O}^{\prime}$ is connected or $\mathfrak{U}=\mathfrak{R}$.

Proof. Since $\mathcal{O}$ is connected, $\mathfrak{R}(\mathcal{O})=m(\mathfrak{F}(\mathcal{O})$ ), where $m$ denotes the average over the compact gauge group. Hence, arguing as in Proposition 2.2, it will suffice to show that $\pi(\mathscr{S}(\mathcal{O}))^{c} \subset \pi\left(\mathfrak{F}^{t} \mid \mathcal{O}^{\prime}\right)^{\prime}$. Now $m\left(\mathfrak{F}^{t} \mid \mathcal{O}^{\prime}\right)=m\left(\mathfrak{F} \mid \mathcal{O}^{\prime}\right)$. If $\mathcal{O}^{\prime}$ is connected this is just $\mathfrak{R} \mid \mathcal{O}^{\prime}$. If $\mathfrak{R}=\mathfrak{A}$, it is contained in $\mathscr{S}(\mathcal{O})$. In either case, it commutes with $\mathscr{S}(\mathcal{O})^{c}$ and the proof is completed by applying the following lemma to $\mathfrak{F}^{t}$ rather than $\mathfrak{F}$. 
2.4. Lemma. Let $\mathcal{O} \in \mathscr{B}$ and $A \in C^{*}(\mathfrak{R})$ such that

then

$$
\begin{aligned}
A m(F) & =m(F) A, & & F \in \mathfrak{F} \mid \mathcal{O}^{\prime} \\
A F & =F A, & & F \in \mathfrak{F} \mid \mathcal{O}^{\prime} .
\end{aligned}
$$

Proof. Given a $\mathscr{G}$-invariant Hilbert space $H_{0}$ in $\mathfrak{F} \mid \mathcal{O}^{\prime}$, we have an associated endomorphism $\varrho_{0}$ with

$$
\psi F=\varrho_{0}(F) \psi, \psi \in H_{0}, F \in C^{*}(\mathfrak{F}) .
$$

By Theorem A.2, it suffices to show $\varrho_{0}(A)=A$. However Theorem A.2 also implies that we can find a sequence of such Hilbert spaces $H_{i} \subset \mathfrak{F}\left(\mathcal{O}_{i}\right)$ equivalent to $H_{0}$ as $\mathscr{G}$-modules, where $\mathcal{O}_{i} \subset \mathcal{O}^{\prime}$ and tends spacelike to infinity. Hence there are gauge invariant unitaries $z_{i j}$ with $z_{i j} H_{j}=H_{i}$ and $z_{i j} \varrho_{j}(F)=\varrho_{i}(F) z_{i j}$ for the associated endomorphisms. Now for any $A \in C^{*}(\mathfrak{R}), \varrho_{0}(A)$ is a limit in norm of $z_{0 i} A z_{0 i}^{*}$ as $i \rightarrow \infty$. Hence if $A$ is as in the statement of the lemma, $\varrho_{0}(A)=A$.

Of course, internal duality is just a $C^{*}$-version of a form of duality proposed by Borchers [14] and termed weak duality in [13]. These results suggest that the concept might deserve a more careful investigation.

We close by pointing out some further situations in which sheaf structures appear. We first consider endomorphisms $\varrho$ of $C^{*}(\mathfrak{U})$ and write $\varrho \in \mathscr{E}$ nd $\mathscr{S}(U)$ if

$$
\varrho(\mathscr{S}(V)) \subset \mathscr{S}(V), \quad V \subset U .
$$

$\varrho, \varrho^{\prime} \in \mathscr{E} n d \mathscr{S}(U)$ imply $\varrho \varrho^{\prime} \in \mathscr{E} n d \mathscr{S}(U)$ and it is easy to check that

$$
\bigcap_{i} \mathscr{E} \text { nd } \mathscr{S}\left(U_{i}\right)=\mathscr{E} \text { nd } \mathscr{S}\left(\bigcup_{i} U_{i}\right) \text {. }
$$

We write $\varrho \in \mathscr{E}$ nd ${ }_{l} \mathscr{S}(U)$ if $\varrho$ is locally normal and

$$
\varrho(A)=A, A \in \mathfrak{X} \mid U .
$$

In other words, $\varrho$ considered as an operation acts trivially on $U$, or $\varrho$ is "localized" in $U^{\prime}$ (cf. $\left[15,\left(1.6^{\prime}\right)\right]$ ). It follows that $\mathscr{E} n d_{l} \mathscr{S}(U) \subset \mathscr{E} n d \mathscr{S}(U)$ and it is again easy to check that

$$
\bigcap_{i} \mathscr{E} n d_{l} \mathscr{S}\left(U_{i}\right)=\mathscr{E} n d_{l} \mathscr{S}\left(\bigcup_{i} U_{i}\right)
$$

Actually it is most useful to turn $\mathscr{E}$ nd $\mathscr{S}(U)$ and $\mathscr{E}$ nd ${ }_{l} \mathscr{S}(U)$ into categories defining $t: \varrho \rightarrow \varrho^{\prime}$ if $\varrho$ and $\varrho^{\prime}$ satisfy (2.5) or (2.7) respectively and $t \in \mathscr{S}(U)$ and satisfies

$$
t \varrho(A)=\varrho^{\prime}(A) t, A \in C^{*}(\mathfrak{U}) .
$$

(2.6) and (2.8) still remain valid in this sense.

Finally the following sheaf structure is used to study superselection sectors in [2]. Let $\mathscr{R} e f \mathfrak{A}(U)$ denote the $W^{*}$-category whose objects are the locally normal representations of $\mathfrak{U}$ and where $T: \pi \rightarrow \pi^{\prime}$ in $\mathscr{R} e f \mathfrak{A}(U)$ if $T: \mathscr{H}_{\pi} \rightarrow \mathscr{H}_{\pi^{\prime}}$ is a bounded linear operator satisfying

$$
T \pi(A)=\pi^{\prime}(A) T, A \in \mathfrak{U} \mid U
$$

Additivity now implies

$$
\bigcap_{i} \mathscr{R} e p \mathfrak{A}\left(U_{i}\right)=\mathscr{R} e p \mathfrak{A}\left(\bigcup_{i} U_{i}\right)
$$




\section{Appendix}

Our analysis of the action of a compact group $\mathscr{G}$ on a field net $\mathfrak{F}$ under the assumptions a)-d) of Sect. 1 is motivated by the discussion for an Abelian group given in the introduction of [9]. It therefore relies on the fundamental analysis of the spectrum condition given by Borchers. We recall two facts about projections in any Hilbert space carrying a representation of the group of space-time translations satisfying the spectrum condition. If $E$ is a projection and $\mathcal{O}$ is an open set then

$$
\bar{E}=\bigvee_{x \in \mathscr{O}} \alpha_{x}(E)
$$

is independent of the choice of $\mathcal{O}$ and is hence $\alpha$-invariant. This is of course the argument of the Reeh-Schlieder theorem [16, Theorem 1]. If $E$ and $F$ are projections with $\left[F, \alpha_{x}(E)\right]=0, x \in \mathcal{O}$ and $F \alpha_{y}(E)=0$ for some $y \in \mathcal{O}$, then $F \alpha_{x}(E)=0$ for all $x$ and hence

$$
\bar{F} \bar{E}=0 \text {. }
$$

This follows from [17, Theorem III.1].

If $\sigma$ and $\tau$ are continuous unitary representations of $\mathscr{G}$ on Hilbert spaces $H_{\sigma}$ and $H_{\tau}$, we define an action of $\mathscr{G}$ on $\mathscr{B}\left(H_{\sigma}, H_{\tau}\right) \otimes \mathfrak{F}(\mathcal{O})$, where the tensor product is the analogue of the tensor product of von Neumann algebras, by requiring

$$
g(S \otimes F)=\tau(g) S \sigma(g)^{*} \otimes g F, S \in \mathscr{B}\left(H_{\sigma}, H_{\tau}\right), F \in \mathfrak{F}(\mathcal{O}) .
$$

The fixed points under this action will be denoted $(\sigma \otimes \mathfrak{F}, \tau \otimes \mathfrak{F})(\mathcal{O})$ or simply $\sigma \mathfrak{F}(\mathcal{O})$ when $\sigma=\tau$. Since $\mathfrak{F}$ is a concrete von Neumann algebra on $\mathscr{H}$, the elements of $(\sigma \otimes \mathfrak{F}, \tau \otimes \mathfrak{F})$ are bounded linear maps from $H_{\sigma} \otimes \mathscr{H}$ to $H_{\tau} \otimes \mathscr{H}$. In any case, the left and right supports of elements of $(\sigma \otimes \mathfrak{F}, \tau \otimes \mathfrak{F})(\mathcal{O})$ lie in $\sigma \mathfrak{F}(\mathcal{O})$ and $\tau \mathfrak{F}(\mathcal{O})$ respectively.

To simplify matters we state our first result for double cones.

A.1. Lemma. Let $\mathcal{O}, \mathcal{O}_{1} \in \mathscr{K}$ with $\overline{\mathcal{O}} \subset \mathcal{O}_{1}$. Then if $\sigma$ is irreducible and $E \in \sigma \mathfrak{F}(\mathcal{O})$ is a non-zero projection, $\bar{E}=I$ and $E \sim I$ in $\sigma \mathfrak{F}\left(\mathcal{O}_{1}\right)$.

Proof. Since $\sigma$ is irreducible and $k$ lies in the centre of $\mathscr{G}$, only the Bose part of $\mathfrak{F}$ contributes to $\sigma \mathfrak{F}$. As $\bar{E}$ is $\alpha$-invariant and $\mathfrak{F}^{-}$is a factor, $\bar{E} \in \mathscr{B}\left(H_{\sigma}\right) \otimes I$. Recalling the definition of $\sigma \mathfrak{F}$, we see that $\bar{E}=I$ since $\sigma$ is irreducible. Next we note that the central support of $E$ in $\sigma \mathfrak{F}\left(\mathcal{O}_{1}\right)$ is the identity since its complement vanishes by (A.2). As $\mathscr{H}$ is separable, the lemma follows if we can show that $E$ and $I$ are properly infinite. Since $1_{\sigma} \otimes \mathfrak{R}(\mathcal{O}) \subset \sigma \mathfrak{F}(\mathcal{O})$ and $\overline{\mathcal{O}} \subset \mathcal{O}_{1}$ it will be enough to know that each $\mathfrak{R}(\mathcal{O})$ is properly infinite (or even infinite). But this follows from [17, Theorem III.3] if we note that the dependence on the additivity assumption is only apparent since we can begin by arguing in terms of a net $\mathfrak{R}_{0}(\mathcal{O})$ defined to be the von Neumann algebra generated by $\alpha_{x}(E), x \in \mathcal{O}$, where $E \neq 0, I$ can be chosen arbitrarily well localized.

We now take $\tau$ to be the identity representation and denote $(\sigma \otimes \mathfrak{F}, \tau \otimes \mathfrak{F})$ by $\mathfrak{F}_{\sigma}$. A priori, we might have $\mathfrak{F}_{\sigma}(\mathcal{O})=0$. If not, taking a polar decomposition, we see that $\mathfrak{F}_{\sigma}(\mathcal{O})$ contains a non-zero partial isometry whose left and right supports are equivalent to $I$ in $\sigma \mathfrak{F}\left(\mathcal{O}_{1}\right)$ and $\mathfrak{R}\left(\mathcal{O}_{1}\right)$ respectively by Lemma A.1. Implementing 
these equivalences we see that $\mathfrak{F}_{\sigma}\left(\mathcal{O}_{1}\right)$ contains a unitary operator. As $\mathfrak{R}\left(\mathcal{O}_{1}\right)$ is properly infinite, $\sigma$ is realized on a $\mathscr{G}$-invariant Hilbert space in $\mathscr{F}\left(\mathcal{O}_{1}\right)$ [9, Proposition 3.6]. Now the set of unitary representations of $\mathscr{G}$ realized in this way is automatically closed under subrepresentations, tensor products, conjugate representations and countable direct sums. Since $\mathscr{G}$ acts faithfully on $\mathfrak{F}(\mathcal{O})$, these representations separate the points of $\mathscr{G}$ and, applying a standard argument using the Stone-Weierstrass theorem (cf. [13, Theorem 3.6]) we have proved:

A.2. Theorem. Let $\mathcal{O}$ be a non-empty open set, then any separable continuous unitary representation of $\mathscr{G}$ can be realized on a $\mathscr{G}$-invariant Hilbert space in $\mathfrak{F}(\mathcal{O})$.

A standard application of the Tannaka duality theorem, cf. the remarks following Theorem 3.7 of [18] or [19, Appendix C] yields

A.3. Corollary. Let $\beta \in$ Aut $\mathfrak{F}(\mathcal{O})$ satisfy $\beta(A)=A$ for gauge-invariant $A$, then $\beta \in \mathscr{G} \mid \mathfrak{F}(\mathcal{O})$.

In Sect. IV of [17], Borchers goes on to consider the ideal structure of the inductive limit of a net of von Neumann algebras. In the same way, we can conclude from Lemma A.1 that $C^{*}(\sigma \mathfrak{F})$ is simple whenever $\sigma$ is irreducible. Hence, in particular, $C^{*}(\mathfrak{R})$ is simple. $C^{*}(\mathfrak{F})$ is also simple since the Bose part is simple and, as an application of Theorem A.2, there is a unitary $\psi \in \mathfrak{F}$ with $k \psi=-\psi$.

Acknowledgements. I gratefully acknowledge the support extended to me by the Istituto Matematico of Rome University and the Centre Universitaire de Marseille-Luminy during the formative stages of this work. I take this opportunity of thanking S. Doplicher and D. Kastler for their hospitality.

\section{References}

1. Haag, R.: Discussion des "axiomes" et des propriétés asymptotiques d'une théorie des champs locale avec particules composées. 75. Colloques Internationaux du CNRS, Lille 1957. Paris: CNRS 1959

2. Roberts, J.E.: New light on the mathematical structure of algebraic field theory. Proc. of Symposia in Pure Mathematics, Vol. 38. Am. Math. Soc. (to appear)

3. Roberts, J.E.: The search for quantum differential geometry. Proceedings of the 6th. International Conference on Mathematical Physics, Berlin 1981. In: Lecture Notes in Physics. Berlin, Heidelberg, New York: Springer (to appear)

4. Buchholz, D., Fredenhagen, K.: Locality and structure of particle states in relativistic quantum theory. Commun. Math. Phys. (to appear)

5. Haag, R., Schroer, B. : Postulates of quantum field theory. J. Math. Phys. 3, 248-256 (1962)

6. Garber, W.-D.: The connexion of duality and causal properties for generalized free fields. Commun. Math. Phys. 42, 195-208 (1975)

7. Borchers, H.J.: Über die Vollständigkeit Lorentzinvarianter Felder in einer zeitartigen Röhre. Nuovo Cimento 19, 787-793 (1961)

8. Glimm, J., Jaffe, A.: Boson quantum field theory models. In: Mathematics of Contemporary Physics, Streater, R.F. (ed.). London, New York: Academic Press 1972

9. Roberts, J.E. : Cross products of von Neumann algebras by group duals. Symp. Math. 20, 335-363 (1976)

10. Driessler, W., Fröhlich, J.: The reconstruction of local observable algebras from the Euclidean Green's functions of relativistic quantum field theory. Ann. Inst. Henri Poincaré A27, 221-236 (1977)

11. Araki, H.: Einführung in die axiomatische Quantenfeldtheorie. Lecture Notes ETH, Zürich 1961/62 (unpublished) 
12. Haag, R., Kadison, R.V., Kastler, D.: Nets of $C^{*}$-algebras and classification of states. Commun. Math. Phys. 16, 81-104 (1970)

13. Doplicher, S., Haag, R., Roberts, J.E.: Fields, observables and gauge transformations. I. Commun. Math. Phys. 13, 1-23 (1969)

14. Borchers, H.J.: Local rings and the connection of spin with statistics. Commun. Math. Phys. 1, 281-307 (1965)

15. Doplicher, S., Haag, R., Roberts, J.E.: Local observables and particle statistics. I. Commun. Math. Phys. 23, 199-230 (1971)

16. Borchers, H.J.: On the converse of the Reeh-Schlieder theorem. Commun. Math. Phys. 10, 269-273 (1968)

17. Borchers, H.J.: A remark on a theorem of B. Misra. Commun. Math. Phys. 4, 315-323 (1967)

18. Roberts, J.E.: Net cohomology and its applications to field theory. In: Quantum Fields-Algebras, Processes, L. Streit, ed., pp. 240-268. Wien, New York: Springer 1980

19. Araki, H., Haag, R., Kastler, D., Takesaki, M.: Extensions of KMS states and chemical potential. Commun. Math. Phys. 53, 97-134 (1977)

Communicated by R. Jost

Received November 27, 1981 